\title{
Trombosis valvular gigante
}

\author{
José Eduardo Ramírez Batista* \\ Alejandro Gutiérrez Fernández* \\ Luis Alonso Pérez*
}

\author{
Omer Leal Fernández** \\ Pedro Azcárate Agüero*
}

\section{Correspondencia}

José Eduardo Ramírez Batista jeramirez47@gmail.com

\footnotetext{
* Departamento de Cardiología. Hospital San Pedro. Logroño. La Rioja. España

** Servicio de Cirugía Cardiovascular. Hospital Viamed Los Manzanos. Logroño. La Rioja. España

Recibido: 31/05/2020

Aceptado: $12 / 06 / 2020$

En línea: $31 / 07 / 2020$
}

Citar como: Ramírez-Batista JE, Gutiérrez-Fernández A, Alonso-Pérez L, Leal-Fernández O, Azcárate-Agüero P. Trombosis valvular gigante. Rev Ecocar Pract (RETIC). 2020 (Jul); 3 (2): 22-25. doi: 10.37615/retic.v3n2a7.

Cite this as: Ramírez-Batista JE, Gutiérrez-Fernández A, Alonso-Pérez L, Leal-Fernández O, Azcárate-Agüero P. Giant valve thrombosis. Rev Ecocar Pract (RETIC). 2020 (Jul); 3 (2): 22-25. doi: 10.37615/retic.v3n2a7.

\section{Palabras clave}

$\triangleright$ Trombosis

$\triangleright$ Ictus

$\triangleright$ Válvula

$\triangleright$ Mecánica

\section{RESUMEN}

Se presenta el caso de un hombre de 63 años con antecedentes de cardiopatía isquémica intervenida, portador de prótesis mecánica mitral, que acude a urgencias por focalidad neurológica. En la ecocardiografía se observa una masa sobre válvula mitral protésica que sugiere la presencia de un trombo protésico. Se inicia anticoagulación con heparina sódica y, ante una evolución desfavorable (el trombo crece), se decide optar por el recambio de la válvula protésica. Tras la intervención se observa en la ecocardiografía de control una prótesis mitral con buena apertura de discos, gradiente medio de 4-5 mmHg y mínima regurgitación intraprotésica.

\section{Presentación del caso}

Varón de 63 años, dislipidémico y fumador con antecedentes de cardiopatía isquémica, enfermedad coronaria crónica de tres vasos revascularizada en junio de 2015 con bypass coronario e implantación de prótesis mitral mecánica ON $X$ 25/33. Acude a urgencias 4 años y medio después por presentar inicio súbito de paresia facial derecha y disartria severa. El paciente ingresó en la planta de Neurología con el diagnóstico de ictus isquémico cerebeloso izquierdo de probable origen cardioembólico. Los controles de INR habían estado consistentemente en rango subterapéutico durante los meses previos.

Durante el ingreso se realiza una ecocardiografía transtorácica (ETT) y posteriormente transesofágica (ETE) donde se objetiva prótesis mitral mecánica bidisco y sobre su anillo posterolateral una masa de unos $6 \mathrm{~mm}$, de refringencia similar al miocardio, que se introduce parcialmente en el espacio del oclusor anterior, impidiendo su cierre completo y que sugiere la presencia de un trombo protésico con un gradiente medio de la prótesis de unos $4 \mathrm{mmHg}$. Además, en la parte más anterior del anillo mitral se observan pequeños filamentos vibrátiles que en el contexto clínico también sugieren trombosis protésica (Figura 1 y Figura 2).

Ante este hallazgo y el alto riesgo de complicaciones valvulares y tromboembólicas se valora el caso de forma conjunta entre los servicios de Neurología, Cardiología y Cirugía Cardíaca (Heart Team). Como el tamaño del trombo no era grande y la prótesis era normofuncionante (a pesar de interferir parcialmente el trombo con el movimiento de la valva anterior), se decidió iniciar perfusión de heparina sódica para mantener unos niveles de TTPA elevados (rango en torno a 3) y se repitió la ETE 8 días después (manejo habitual en la trombosis valvular protésica no obstructiva).

A pesar del tratamiento con heparina sódica se observó una progresión rápida del trombo que había crecido considerablemente y provocaba inmovilidad del disco anterior (Vídeo 1 a Vídeo 4). El gradiente medio de la prótesis mitral era de $9,6 \mathrm{mmHg}$ (Vídeo 5) y el orificio de apertura mitral es de $0,5 \mathrm{~cm}^{2}$ (Figura 3). Debido al gran volumen del trombo, a que interfería con el funcionamiento de la válvula mitral y a su refractariedad al ciclo inicial con heparina sódica se decidió intervenir quirúrgicamente al paciente. 
Se realizó una intervención quirúrgica programada objetivando trombosis de prótesis mitral mecánica de gran tamaño (Figura 4 y Vídeo 6). Se procedió al recambio de la prótesis mitral trombosada. El postoperatorio cursó sin incidencias. En la ecocardiografía al alta se observó prótesis mitral mecánica con buena apertura de discos, gradiente medio 4-5 mmHg y mínima regurgitación intraprotésica.

\section{Estudio por imagen}

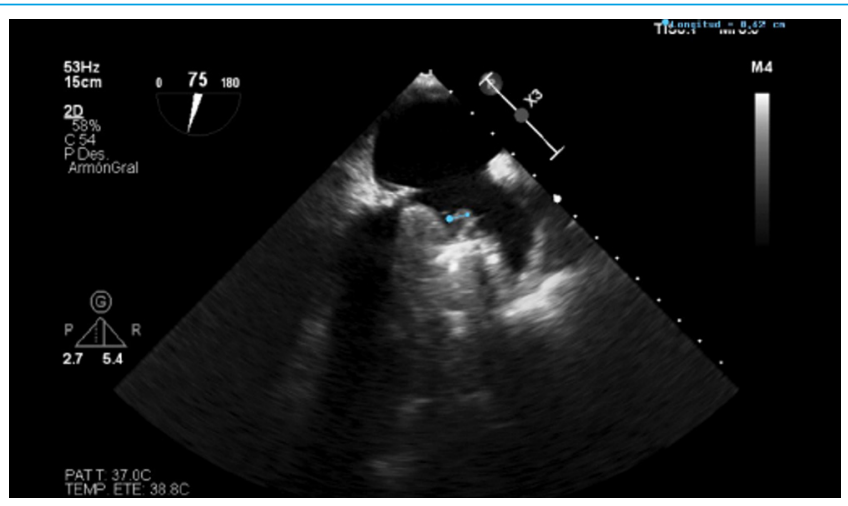

Figura 1. ETE bidimensional, plano esófago medio a $75^{\circ}$, donde se observa una masa de unos $6 \mathrm{~mm}$ en prótesis mitral mecánica bidisco (línea azul)

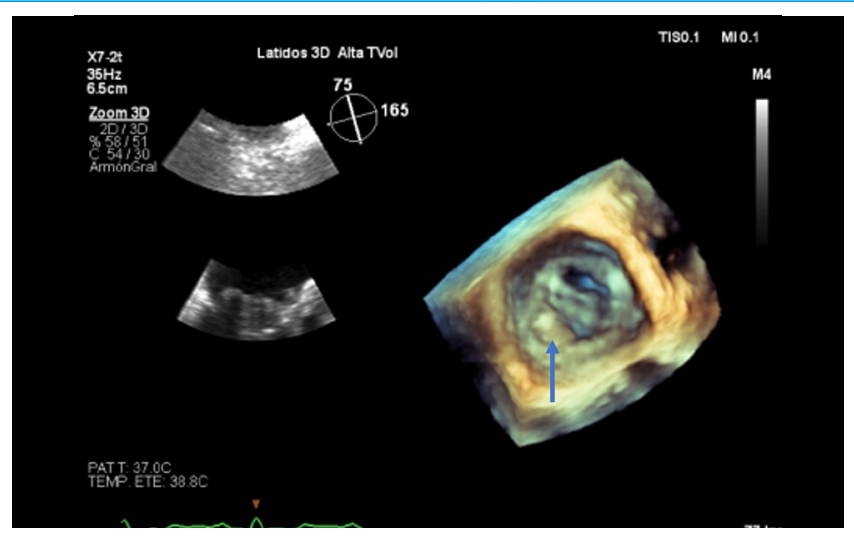

Figura 2. ETE tridimensional en donde se observa sobre el anillo una masa que se introduce parcialmente en el espacio del oclusor anterior, impidiendo el cierre completo de la válvula y que sugiere la presencia de un trombo

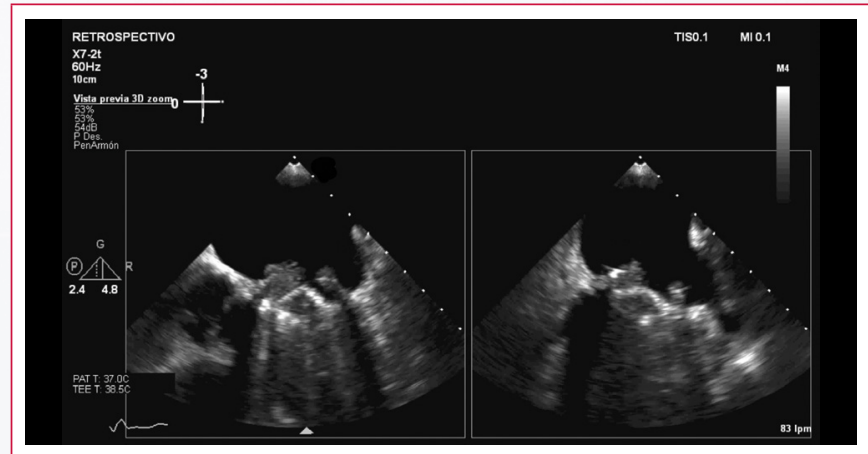

Vídeo 1. ETE bidimensional, plano esófago medio 0,$X$ plane. Se observa trombosis masiva de la valva anterior mitral protésica que produce una inmovilidad de dicho disco. El otro disco también presentaba material trombótico pero su movilidad estaba conservada

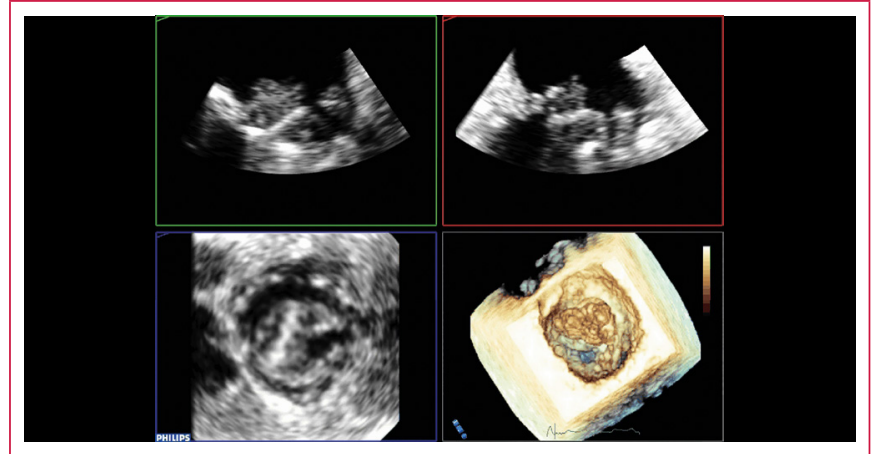

Vídeo 2. Ecocardiografía tridimensional, reconstrucción MPR (visión desde la aurícula orientada). Se observa el gran trombo sobre la valva anterior mitral

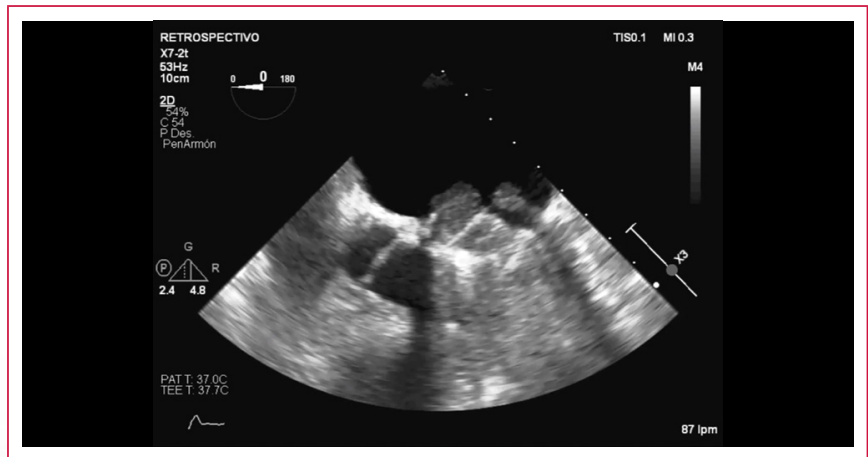

Vídeo 3. Varios vídeos de distintos planos ecocardiográficos, primero de cuatro cámaras sin color, después TSVI sin color, luego cuatro cámaras con color, donde se observa que sólo se abre la válva mitral posterior. Y finalmente reconstrucción tridimensional MPR (visión orientada)

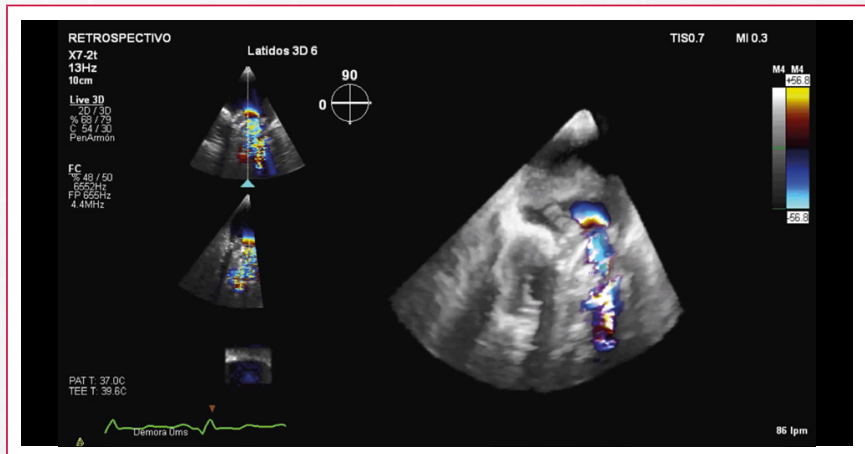

Vídeo 4. Varios vídeos tridimensionales que muestran que sólo se abría la valva mitral posterior y el gran tamaño del trombo protésico

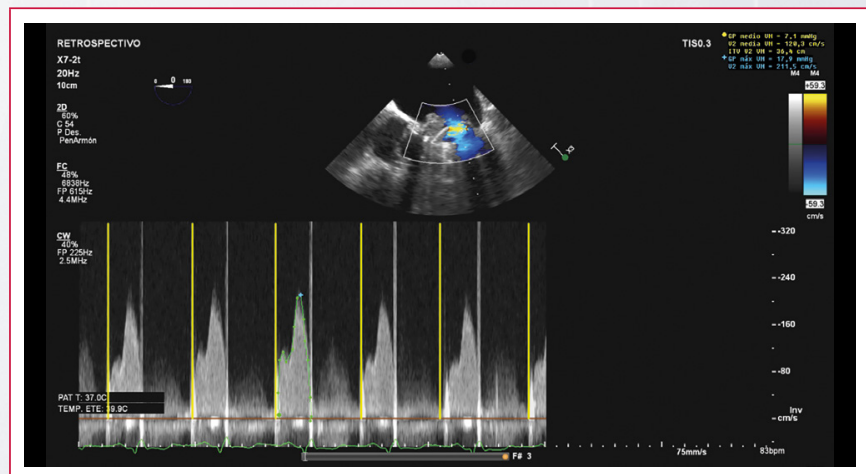

Vídeo 5. Doppler continuo mitral. En amarillo se destaca cómo por Doppler continuo puede observarse la falta de movilidad de un disco. Es decir, no hay señal de apertura del disco mitral anterior 


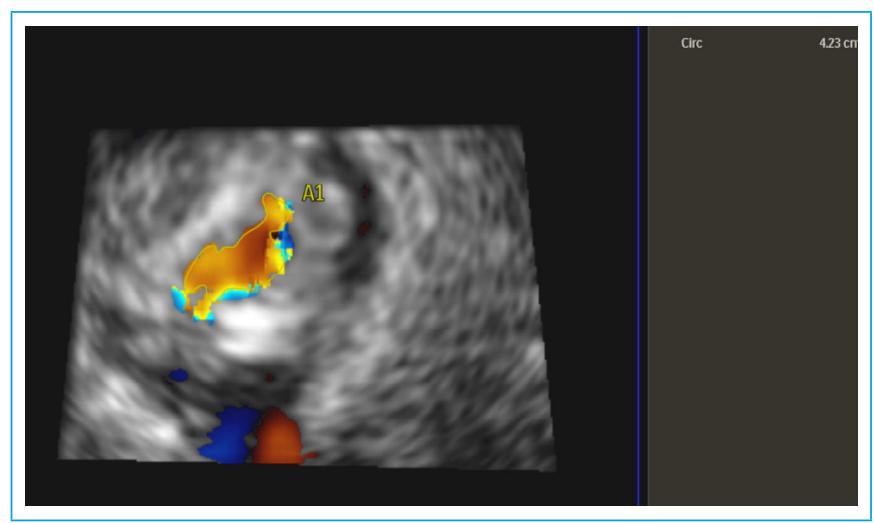

Figura 3. Planimetría tridimensional del área de apertura del disco mitral posterior mediante Doppler color

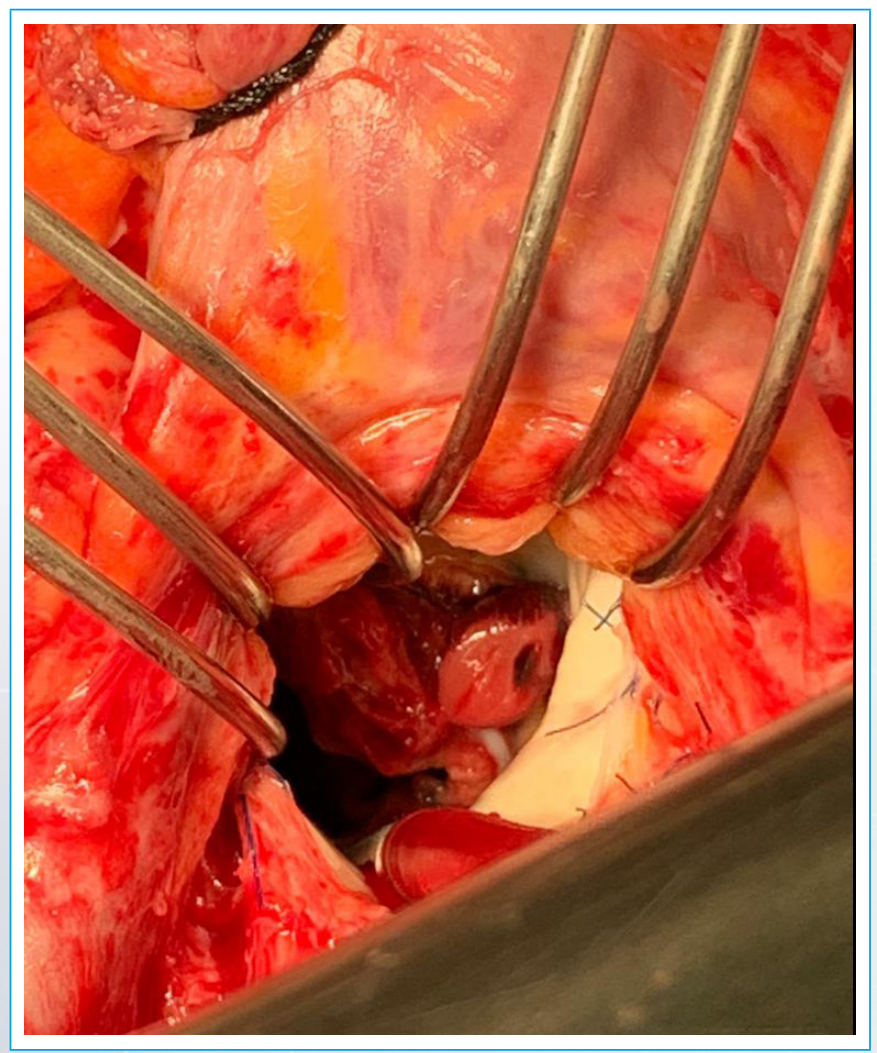

Figura 4. Foto quirúrgica del gran trombo que presentaba el paciente sobre la valva mitral anterior

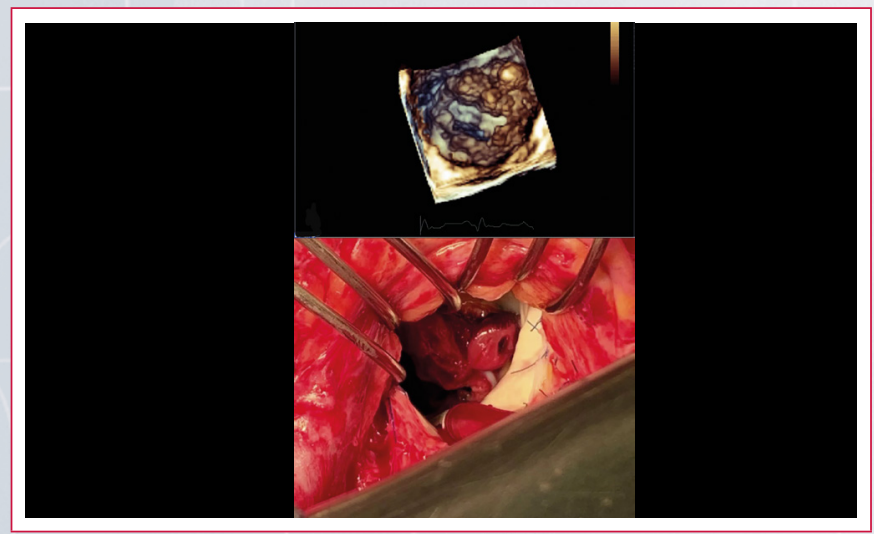

Vídeo 6. Arriba, reconstrucción tridimensional por ETE; abajo, foto quirúrgica del trombo mitral

\section{Discusión}

La trombosis de prótesis valvulares (TPV) es una de las complicaciones asociadas a las prótesis como son la obstrucción, eventos embólicos, sangrado, insuficiencia valvular, endocarditis infecciosa y hemólisis. Los trombos valvulares pueden ser obstructivos o no obstructivos y pueden o no provocar eventos tromboembólicos. El riesgo de trombosis aumenta en válvulas mecánicas, en posiciones mitral y tricúspide y en casos de anticoagulación subóptima ${ }^{(1)}$.

La incidencia documentada de TPV es de 0,3-1,3\% por año, observándose tasas de hasta el $6 \%$ en los pacientes infracoagulados, aunque su incidencia real se desconoce y tiende a ser subestimada, ya que puede estar presente de manera silente. Algunos estudios estiman que ésta afecta hasta un tercio de los portadores de prótesis ${ }^{(2)}$. En pacientes con válvulas mecánicas en tratamiento anticoagulante, la incidencia de embolización sistémica sintomática es de aproximadamente $2,5-5,7 \%{ }^{(3)}$.

Las manifestaciones clínicas dependen del grado de disfunción valvular presente y pueden ir desde cambios en el click de cierre de la prótesis y aumento en el gradiente transprotésico, hasta fenómenos tromboembólicos (pulmonar, cerebral), insuficiencia cardíaca, síncope y muerte súbita ${ }^{(3)}$. Estos signos y síntomas pueden ocurrir ante la presencia de obstrucción valvular o en su ausencia.

El tratamiento de la trombosis valvular protésica no obstructiva (TVPNO) consiste en la optimización de la anticoagulación, que consigue su resolución en hasta el $72 \%$ de los pacientes durante la hospitalización con desaparición completa y progresiva con el tiempo. Según las guías ESC 2017, los pacientes con trombos de gran tamaño (> $10 \mathrm{~mm}$ ) y fenómenos embólicos deben ser intervenidos quirúrgicamente, dejando la fibrinólisis como alternativa para aquellos pacientes con alto riesgo quirúrgico. En casos en los que haya trombos de gran tamaño sin eventos tromboembólicos se debe optimizar la anticoagulación y, si hay persistencia del trombo, se planteará la cirugía o fibrinólisis ${ }^{(4)}$.

\section{Conclusiones}

La trombosis valvular protésica no obstructiva (TVPNO) es una entidad de alto riesgo que conlleva una importante morbimortalidad con aparición de complicaciones en un $27,6 \%$ durante la hospitalización y en un 59,5\% tras el alta. La anticoagulación subóptima es el principal factor de riesgo y su optimización es efectiva para el tratamiento en la mayoría de los casos. Aquellos pacientes con trombos $\geq 10 \mathrm{~mm}$ o que no tienen una resolución progresiva posterior a la intensificación del tratamiento, se benefician de cirugía o fibrinólisis dependiendo de sus comorbilidades y su estado clínico. Hasta la fecha no se han realizado ensayos clínicos controlados, por lo que el manejo óptimo sigue siendo controvertido.

\section{Ideas para recordar}

- La trombosis valvular protésica, tanto obstructiva como no obstructiva, es una complicación que requiere un diagnóstico y tratamiento inmediatos.

- Los factores de riesgo son disfunción ventricular, eventos embólicos previos, estados de hipercoagulabilidad, posición mitral o tricúspide y anticoagulación subóptima.

- Aunque la mayoría de los casos de TVPNO cursan de manera asintomática, algunos pueden debutar con eventos embólicos o progresar a la obstrucción valvular. 
- La optimización de la anticoagulación es efectiva en la mayoría de los casos de TVPNO excepto en aquellos trombos $\geq 10 \mathrm{~mm}$ en los que el recambio valvular es de elección.

- En pacientes de alto riesgo quirúrgico se puede plantear la fibrinólisis como alternativa terapéutica a la cirugía.

\section{Bibliografía}

1. Roudaut R, Serri K, Lafitte S. Thrombosis of prosthetic heart valves: diagnosis and therapeutic considerations. Heart 2007; 93: 137-142.
2. Laplace $G$, Lafitte $S$, Labèque JN, et al. Clinical, significance of early thrombosis after prosthetic mitral valve replacement. J Am Coll Cardiol 2004; 43: 1283-1290.

3. Dangas GD, Weitz Jl, Giustino G, et al. Prosthetic heart valve thrombosis. J Am Coll Cardiol 2016; 68: 2670-2689.

4. Baumgartner H, Falk V, Bax JJ, et al. ESC Scientific Document Group, 2017 ESC/EACTS Guidelines for the management of valvular heart disease. Eur Heart J 2017; 38: 2739-2791. 